\title{
Application of the Aboodh Transform for Solving Fractional Delay Differential Equations
}

\author{
Mohamed Elarbi Benattia ${ }^{1 *}$ and Kacem Belghaba ${ }^{2}$ \\ ${ }^{1}$ High school of economics- Oran , Laboratory of Mathematics and its Applications (LAMAP), Algeria \\ ${ }^{2}$ University of Oran 1, Ahmed Ben Bella, Laboratory of Mathematics and its Applications (LAMAP), Algeria \\ ${ }^{*}$ Corresponding author
}

\section{Article Info}

Keywords: Aboodh Transform, Fractional Delay differential Equation, Partial derivatives, function series.

2010 AMS: 65D15, 65L05

Received: 11 March 2020

Accepted: 13 July 2020

Available online: 29 September 2020

\begin{abstract}
In this article, we extend the concept of the Aboodh transform to the solution of partial differential equations of fractional order using Caputo's fractional derivative. The transformation concerned is applicable to solve many classes of partial differential equations with derivatives of order and integrals. Consequently, the fractional Delay Differential Equations (DDEs) which we are going to study in this work. The resulting numerical proofs show that the method converges favorably towards the analytical solution.
\end{abstract}

\section{Introduction}

Many physical problems can be described by mathematical models that involve ordinary or partial differential equations. A mathematical model is a simplified description of physical reality expressed in mathematical terms. Thus, the investigation of the exact or approximation solution helps us to understand the means of these mathematical models. Several numerical methods were developed for solving ordinary or partial differential equations. the Aboodh transform method is used to solve the linear and nonlinear fractional delay differential equations (FDDEs). New integral transform Aboodh transform is particularly useful for finding solutions for fractional delay differential equation. Aboodh transform is a useful technique for solving these equations but this transform is totally incapable of handling nonlinear equations because of the difficulties that are caused by the nonlinear terms. [1,3-7]. Fractional delay differential equations (FDDEs) are a very recent topic. Although it seems natural to model certain processes and systems in engineering and other science (with memory and heritage properties) with this kind of equations, only in the last few years has the attention of the scientific community been devoted to them. The purpose of this work is to find the approximate solution of delay differential equations of fractional order using aboodh transform and adomian decomposition methods [9].

This work consists of three sections, as well as, this introduction. In Section 2, definition and properties of the Aboodh transform and method of solution is presented. Section 3, basic concepts of delay differential equations and fractional calculus are given. Finally, the application of the Aboodh transform method for solving delay differential equations of fractional order, with illustrative examples have been given.

\section{The Aboodh Transform}

The Aboodh transform is a new integral transform similar to the Laplace transform and other integral transforms that are defined in the time domain $t \geq 0$, such as the Sumudu transform, the Natural transform and the Elzaki transform, respectively [4].

An Aboodh transform is defined for functions of exponential order. We consider functions in the set $F$ defined by:

$$
F=\left\{f ( t ) : | f ( t ) | < M e ^ { - v t } , \text { if } t \in \left[0 ; \infty\left[, M, k_{1}, k_{2}>0 ; k_{1} \leq v \leq k_{2}\right\}\right.\right.
$$

For a given function in the set $F$, the constant $M$ must be finite number and $k_{1}, k_{2}$ may be infinite or finite with variable $v$ define as $k_{1} \leq v \leq k_{2}$. 


\begin{tabular}{|c|c|}
\hline$f(t)$ & $T(v)=A[f(t)]$ \\
\hline 1 & $\frac{1}{v^{2}}$ \\
\hline$t$ & $\frac{1}{v^{3}}$ \\
\hline$t^{n}, n \geq 1$ & $\frac{n !}{v^{n+2}}$ \\
\hline$e^{a t}$ & $\frac{1}{v^{2}-a v}$ \\
\hline $\sin (a t)$ & $\frac{1}{v\left(v^{2}+a^{2}\right)}$ \\
\hline $\cos (a t)$ & $\frac{1}{v^{2}+a^{2}}$ \\
\hline $\sinh (a t)$ & $\frac{1}{v\left(v^{2}-a^{2}\right)}$ \\
\hline$t \cosh (a t)$ & $\frac{1}{v^{2}-a^{2}}$ \\
\hline
\end{tabular}

Table 1: Aboodh transform of some functions

Then, the Aboodh integral transform denoted by the operator $A(:)$ is defined by the integral equation:

$$
T(v)=A[f(t)]=\frac{1}{v} \int_{0}^{\infty} f(t) e^{-v t} d t, \quad t \geq 0, k_{1} \leq v \leq k_{2} .
$$

Standard Aboodh transform for some special functions found are given below in Table 1 .

Theorem 2.1. Aboodh transform of some partial derivatives :

$$
\begin{aligned}
& (i)-A\left[f^{\prime}(t)\right]=v T(v)-\frac{f(0)}{v}, \\
& (i i)-A\left[f^{\prime \prime}(t)\right]=v^{2} T(v)-\frac{f^{\prime}(0)}{v}-f(0), \\
& (\text { iii })-T^{n}(v)=A\left[f^{(n)}(t)\right]=v^{n} T(v)-\sum_{k=0}^{n-1} \frac{f^{(k)}(0)}{v^{2-n+k}} .
\end{aligned}
$$

Remark 2.2. The Aboodh transform is linear, i.e., if $\alpha$ and $\beta$ are any constants and $f(t)$ and $g(t)$ are functions defined over the set $F$ above, then :

$$
A[\alpha f(t)] \pm \beta g(t)]=\alpha A[f(t)] \pm \beta A[g(t)] .
$$

\subsection{Aboodh transform method}

Let us consider the general nonlinear ordinary differential equation (ODE) of the form [8]:

$$
\frac{d^{n} y(t)}{d t^{n}}+P(y)+Q(t-\tau)=g(t), n=1,2,3, \ldots .
$$

with initial condition:

$$
y^{(k)}(0)=y_{0}^{k},
$$

where $\frac{d^{n} y}{d t^{n}}$ is the derivative of $y$ of order $n, P$ is the linear bounded operator, $Q$ is a nonlinear bounded operator and $g(t)$ is a given continuous function, and $y=y(t)$.

Inspired by Wu [4] method, if we take Abooth transform on both sides of Eqs((2.3)-(2.4)), the linear part with constant coefficients is then transferred into an algebraic one, so that we can identify the Lagrange multiplier in a more straightforward way. Now, we extend this idea to find the unknown Lagrange multiplier. Taking the above Aboodh transform to both sides of eq(2.3), and (2.2), then the linear part is transformed into an algebraic equation as follows:

Applying the Aboodh transform, we obtain

$$
A\left[\frac{d^{n} y(t)}{d t^{n}}\right]+A[P(y)]+A[Q(t-\tau)]=A[g(t)]
$$

But

$$
A\left[\frac{d^{n} y(t)}{d t^{n}}\right]=v^{n} A(y(t))-\frac{E}{v^{2-n+k}}
$$


where $E=\sum_{k=0}^{n-1} g^{(k)}(0)$,

$$
A(y(t))=\frac{E}{v^{2+k}}-v^{-n} A[P(y)]-v^{-n} A[Q(t-\tau)]+v^{-n} A[g(t)] .
$$

The standard Aboodh decomposition method defines the solution $y(t)$ by the series:

$$
y(t)=\sum_{n=0}^{\infty} y_{n}(t)
$$

the nonlinear operator is decomposed as:

$$
Q(t-\tau)=\sum_{n=0}^{\infty} B_{n}
$$

where $B_{n}$ is the a domain polynomial of $y_{0}, y_{1}, y_{2}, \ldots \ldots, y_{n}$ that are given by:

$$
B_{n}=\frac{1}{n !} \frac{d^{n}}{d \lambda^{n}}\left[Q\left(\sum_{n=0}^{\infty} \lambda^{n} y_{n}\right)\right]
$$

then the Adomian series reads :

$$
\left\{\begin{array}{c}
B_{0}=f\left(y_{0}\right) \\
B_{1}=y_{1} f^{\prime}\left(y_{0}\right) \\
B_{2}=y_{2} f^{\prime}\left(y_{0}\right)+\frac{1}{2} y_{1}^{2} f^{\prime \prime}\left(y_{0}\right) \\
B_{3}=y_{3} f^{\prime}\left(y_{0}\right)+y_{1} y_{2} f^{\prime \prime}\left(y_{0}\right)+\frac{1}{3 !} y_{1}^{3} f^{\prime \prime \prime}\left(y_{0}\right) \\
\vdots
\end{array}\right.
$$

Applying (2.8) and (2.9) into (2.7), we obtain

$$
A\left(\sum_{n=0}^{\infty} y_{n}\right)=\frac{E}{v^{2+k}}-v^{-n} A\left[P\left(\sum_{n=0}^{\infty} y_{n}\right)\right]-v^{-n} A\left[\sum_{n=0}^{\infty} B_{n}\right]+v^{-n} A[g(t)],
$$

comparing both side of (2.11):

$$
\begin{aligned}
& A\left[y_{0}\right]=\frac{E}{v^{2+k}}+v^{-n} A[g(t)], \\
& A\left[y_{1}\right]=-v^{-n} A\left[P y_{0}\right]-v^{-n} A\left[B_{0}\right], \\
& A\left[y_{2}\right]=-v^{-n} A\left[P y_{1}\right]-v^{-n} A\left[B_{1}\right] .
\end{aligned}
$$

In general the recursive relation is given by:

$$
A\left[y_{n}\right]=-v^{-n} A\left[P y_{n-1}\right]-v^{-n} A\left[B_{n-1}\right], \quad n \geqslant 1
$$

By the Aboodh transformed inverse method to(2.12)-(2.15), we get :

$$
\begin{aligned}
& y_{0}=K(t) \\
& y_{n}=-A^{-1}\left[v^{-n} A\left[P y_{n-1}\right]\right]-A^{-1}\left[v^{-n} A\left[B_{n-1}\right]\right], \quad n \geqslant 1 .
\end{aligned}
$$

where $K(t)$ is a function that satisfies the initial conditions. 


\subsection{Illustrative examples}

Example 2.3. Consider the following nonlinear delay differential equation (NDDE) of first order:

$$
y^{\prime}(t)=1+2 y^{2}\left(\frac{t}{2}\right) \quad, 0 \leq t \leq 1,
$$

with the initial condition $y(0)=0$. The exact solution of the problem is :

$$
y(t)=\sinh (t) .
$$

Applying the Aboodh transform on both sides, we have:

$$
A\left[y^{\prime}(t)\right]=A[1]+2 A\left[y^{2}\left(\frac{t}{2}\right)\right],
$$

By definition (ii) in theorem (2.1), we have :

$$
A\left[y^{\prime}(t)\right]=v T(v)-\frac{f(0)}{v}=\frac{1}{v^{2}}+2 A\left[f^{2}\left(\frac{t}{2}\right)\right] .
$$

So, we have :

$$
T(v)=A(y(t))=\frac{1}{v^{3}}+\frac{2}{v} A\left[y^{2}\left(\frac{t}{2}\right)\right] .
$$

Applying the Aboodh inverse operator, $A^{-1}$ on both sides of (2.17), we obtain:

$$
y(t)=A^{-1}\left[\frac{1}{v^{3}}\right]+2 A^{-1}\left[\frac{1}{v} A\left[y^{2}\left(\frac{t}{2}\right)\right]\right] .
$$

Using the Table 1, we have: $A^{-1}\left[\frac{1}{v^{3}}\right]=t$, hence:

$$
y_{0}(t)=A^{-1}\left[\frac{1}{v^{3}}\right]=t,
$$

so,

$$
\begin{aligned}
& y_{0}\left(\frac{t}{2}\right)=\frac{t}{2} \\
& y_{n+1}=2 A^{-1}\left[\frac{1}{v} A\left[B_{n}\right]\right],
\end{aligned}
$$

from equation (2.10), we have :

$$
\left\{\begin{array}{c}
B_{0}=f\left(y_{0}\right)=y_{0}^{2}\left(\frac{t}{2}\right)=\frac{t^{2}}{4} \\
B_{1}=y_{1} f^{\prime}\left(y_{0}\right)=2 y_{0}\left(\frac{t}{2}\right) y_{1}\left(\frac{t}{2}\right) \\
B_{2}=y_{2}\left(\frac{t}{2}\right) 2 y_{0}\left(\frac{t}{2}\right)+\frac{1}{2} y_{1}^{2} \\
\vdots
\end{array}\right.
$$

For $n=0$, the equation (2.19), become :

$$
y_{1}(t)=2 A^{-1}\left[\frac{1}{v} A\left[B_{0}\right]\right]=2 A^{-1}\left[\frac{1}{v} A\left[\frac{t^{2}}{4}\right]\right]=2 A^{-1}\left[\frac{1}{2 v^{5}}\right]=\frac{t^{3}}{3 !}
$$

So,

$$
y_{1}\left(\frac{t}{2}\right)=\frac{t^{3}}{48} .
$$

For $n=1$, the equation (2.19), become :

$$
y_{2}(t)=2 A^{-1}\left[\frac{1}{v} A\left[B_{1}\right]\right]=2 A^{-1}\left[\frac{1}{v} A\left[2 y_{0}\left(\frac{t}{2}\right) y_{1}\left(\frac{t}{2}\right)\right]\right]=2 A^{-1}\left[\frac{1}{v} A\left[\frac{t^{4}}{48}\right]\right]=\frac{t^{5}}{5 !}
$$

So,

$$
y_{2}\left(\frac{t}{2}\right)=\frac{t^{5}}{3840} .
$$

For $n=2$, the equation (2.19), become:

$$
\begin{aligned}
& y_{3}(t)=2 A^{-1}\left[\frac{1}{v} A\left[B_{2}\right]\right]=2 A^{-1}\left[\frac{1}{v} A\left[2 y_{2}\left(\frac{t}{2}\right) y_{0}\left(\frac{t}{2}\right)+y_{1}^{2}\left(\frac{t}{2}\right)\right]\right] \\
& =2 A^{-1}\left[\frac{1}{v} A\left[\frac{t^{6}}{3840}+\frac{t^{6}}{2304}\right]\right]=2 A^{-1}\left[\frac{1}{v}\left[\frac{6 !}{3840 v^{8}}+\frac{6 !}{2304 v^{8}}\right]\right]=\frac{t^{7}}{7 !} .
\end{aligned}
$$

Therefore the approximate solution is given as:

$$
y(t)=y_{0}(t)+y_{1}(t)+y_{2}(t)+\ldots=t+\frac{t^{3}}{3 !}+\frac{t^{5}}{5 !}+\frac{t^{7}}{7 !} \ldots \ldots=\sinh (t) .
$$


Example 2.4. Consider the following linear delay differential equation (NDDE) of first order:

$$
y^{\prime}(t)=e^{t} y\left(\frac{t}{2}\right)+y(t) \quad, 0 \leq t \leq 1
$$

with the initial condition $y(0)=1$.

The exact solution of the problem is :

$$
y(t)=e^{2 t}
$$

Applying the Aboodh transform on both sides, we have :

$$
A\left[y^{\prime}(t)\right]=A\left[e^{t} y\left(\frac{t}{2}\right)+y(t)\right] .
$$

By definition (ii) in theorem (2.1), we have :

$$
\begin{aligned}
& v A[y(t)]-\frac{y(0)}{v}=A\left[e^{t} y\left(\frac{t}{2}\right)+y(t)\right], \\
& A[y(t)]=\frac{1}{v^{2}}+\frac{1}{v} A\left[e^{t} y\left(\frac{t}{2}\right)+y(t)\right] .
\end{aligned}
$$

Applying the Aboodh inverse operator, $A^{-1}$ on both sides of (2.20), we obtain :

$$
y(t)=A^{-1}\left[\frac{1}{v^{2}}\right]+A^{-1}\left[\frac{1}{v} A\left[e^{t} y\left(\frac{t}{2}\right)+y(t)\right]\right] .
$$

By the Aboodh transform method, equation (2.21), can be written as :

$$
\begin{gathered}
y_{0}(t)=1 \\
y_{n+1}(x)=A^{-1}\left[\frac{1}{v} A\left[e^{t} y_{n}\left(\frac{t}{2}\right)+y_{n}(t)\right]\right], n \geq 0 .
\end{gathered}
$$

For $n=0$, we have :

$$
y_{1}(t)=A^{-1}\left[\frac{1}{v} A\left[e^{t} y_{0}\left(\frac{t}{2}\right)+y_{0}(t)\right]\right]=A^{-1}\left[\frac{1}{v}\left[A\left[e^{t}\right]+A[1]\right]\right] .
$$

But,

$$
A\left[e^{t}\right]=\frac{1}{v^{2}-v}=\frac{1}{v^{2}}\left(\frac{1}{1-\frac{1}{v}}\right)=\frac{1}{v^{2}}\left(1+\frac{1}{v}+\frac{1}{v^{2}}+\frac{1}{v^{3}}+\ldots .\right)=\frac{1}{v^{2}}+\frac{1}{v^{3}}+\frac{1}{v^{4}}+\ldots
$$

Hence, equation (2.22), can be written as:

$$
y_{1}(t)=A^{-1}\left[\frac{1}{v^{3}}+\frac{1}{v^{4}}+\frac{1}{v^{5}}+\ldots \ldots \ldots+\frac{1}{v^{3}}\right]=2 t+\frac{t^{2}}{2 !}+\frac{t^{3}}{3 !}+\frac{t^{4}}{4 !}+\ldots \ldots
$$

we obtain the following approximation for $n \geq 1$ :

$$
\begin{aligned}
& y_{2}(t)=\frac{3 t^{2}}{2}+\frac{5 t^{3}}{12}+\frac{11 t^{4}}{24} .+\ldots \ldots . \\
& y_{3}(t)=\frac{9 t^{3}}{2}+\frac{15 t^{4}}{128}+\frac{259 t^{5}}{1920}+\ldots \ldots
\end{aligned}
$$

Thus the approximate becomes :

$$
\begin{aligned}
& y(t)=y_{0}(t)+y_{1}(t)+y_{2}(t)+y_{3}(t)+\ldots \\
& =1+2 t+2 t^{2}+\frac{8 t^{3}}{3 !}+\frac{16 t^{4}}{4 !}+\ldots \ldots \ldots \ldots=e^{2 t} .
\end{aligned}
$$

\section{Fractional Delay Differential Equation}

In this section we apply the Aboodh decomposition method to solve linear and nonlinear fractional delay differential equation.

Definition 3.1. The Aboodh transform of the Caputo fractional derivative is defined as follows [2]:

$$
\begin{gathered}
i)-A\left[D^{\alpha} f(t)\right]=v^{\alpha} A[f(t)]-\sum_{k=0}^{n-1} v^{\alpha-2-k} f^{(k)}(0), \quad n-1<\alpha \leqslant n . \\
\text { ii })-A\left(t^{\alpha}\right)=\frac{\Gamma(\alpha+1)}{v^{\alpha+2}} .
\end{gathered}
$$




\subsection{Analysis of the method}

Let us consider the general nonlinear ordinary differential equation of the form:

$$
D^{\alpha} y(t)+P(y)+Q(t-\tau)=g(t), \quad \tau \in \mathbb{R}, \quad t<\tau, \quad n-1<\alpha \leqslant n .
$$

With initial condition:

$$
y^{(k)}(0)=y_{0}^{k},
$$

where $D^{\alpha} y(t)$ is the term of the fractional order derivative , $P$ is the linear bounded operator, $Q$ is a nonlinear bounded operator and $g(t)$ is a given continuous function, and $y=y(t)$.

The Aboodh decomposition method consists of applying the Aboodh transform first on both side of(3.1), to give:

$$
A\left[D^{\alpha} y(t)\right]+A[P(y)]+A[Q(t-\tau)]=A[g(t)],
$$

by definition (3.1)

$$
A(y(t))=\frac{E}{v^{2+k}}-v^{-\alpha} A[P(y)]-v^{-\alpha} A[Q(t-\tau)]+v^{-\alpha} A[g(t)],
$$

where $E=\sum_{k=0}^{n-1} g^{(k)}(0)$.

The standard Aboodh decomposition method defines the solution $y(t)$ by the series:

$$
y(t)=\sum_{n=0}^{\infty} y_{n}(t),
$$

the nonlinear operator is decomposed as:

$$
Q(t-\tau)=\sum_{n=0}^{\infty} B_{n}
$$

Where $B_{n}$ as in (2.9). The first a domain polynomials are given as in (2.10). Apply (3.3) and (3.4) in (3.2), we have:

$$
A\left[\sum_{n=0}^{\infty} y_{n}(t)\right]=\frac{E}{v^{2+k}}-v^{-\alpha} A\left[P \sum_{n=0}^{\infty} y_{n}(t)\right]-v^{-\alpha} A\left[\sum_{n=0}^{\infty} B_{n}\right]+v^{-\alpha} A[g(t)] .
$$

Comparing both side of (3.5):

$$
\begin{aligned}
& A\left[y_{0}\right]=\frac{E}{v^{2+k}}+v^{-\alpha} A[g(t)], \\
& A\left[y_{1}\right]=-v^{-\alpha} A\left[P y_{0}\right]-v^{-\alpha} A\left[B_{0}\right], \\
& A\left[y_{2}\right]=-v^{-\alpha} A\left[P y_{1}\right]-v^{-\alpha} A\left[B_{1}\right] . .
\end{aligned}
$$

In general the recursive relation is given by:

$$
A\left[y_{n}\right]=-v^{-\alpha} A\left[P y_{n-1}\right]-v^{-\alpha} A\left[B_{n-1}\right], \quad n \geqslant 1,
$$

applying inverse Aboodh transform to(3.6)-(3.8), then:

$$
\begin{aligned}
& y_{0}=K(t), \\
& y_{n}=-A^{-1}\left[v^{-\alpha} A\left[P y_{n-1}\right]\right]-A^{-1}\left[v^{-\alpha} A\left[B_{n-1}\right]\right], \quad n \geqslant 1,
\end{aligned}
$$

where $K(t)$ is a function that satisfies the initial conditions.

Example 3.2. Consider the nonlinear delay differential equation of first order:

$$
D^{\alpha} y(t)=1+2 y^{2}\left(\frac{t}{2}\right), \quad 0 \leqslant t \leqslant 1, \quad 0<\alpha \leqslant 1 .
$$

with initial condition:

$$
y(0)=0,
$$

apply Aboodh transform to both side of equation(3.9), we obtain :

$$
A\left[D^{\alpha} y(t)\right]=A\left[1+2 y^{2}\left(\frac{t}{2}\right)\right]
$$


by using definition (3.1) and initial condition(3.10), we get :

$$
\begin{aligned}
& v^{\alpha} A[y(t)]=\frac{1}{v^{2}}+A\left[2 y^{2}\left(\frac{t}{2}\right)\right], \\
& A[y(t)]=\frac{1}{v^{2+\alpha}}+\frac{1}{v^{\alpha}} A\left[2 y^{2}\left(\frac{t}{2}\right)\right] .
\end{aligned}
$$

Applying the inverse Aboodh transform to (3.11), we obtain :

$$
y(t)=A^{-1}\left[\frac{1}{v^{2+\alpha}}\right]+A^{-1}\left[\frac{1}{v^{\alpha}} A\left[2 y^{2}\left(\frac{t}{2}\right)\right]\right],
$$

where,

$$
y_{0}(t)=A^{-1}\left[\frac{1}{v^{2+\alpha}}\right]=\frac{t^{\alpha}}{\Gamma(\alpha+1)} .
$$

So, we have :

$$
y_{0}\left(\frac{t}{2}\right)==\frac{t^{\alpha}}{2^{\alpha} \Gamma(\alpha+1)} .
$$

And,

$$
y_{n+1}(t)=A^{-1}\left[\frac{1}{v^{\alpha}} A\left[2 B_{n}\right]\right] .
$$

From equation (2.10), we have:

$$
\begin{gathered}
B_{0}=y_{0}^{2}\left(\frac{t}{2}\right) \\
B_{1}=2 y_{0}\left(\frac{t}{2}\right) y_{1}\left(\frac{t}{2}\right) \\
B_{2}=y_{2}\left(\frac{t}{2}\right) 2 y_{0}\left(\frac{t}{2}\right)+\frac{1}{2} y_{1}^{2} .
\end{gathered}
$$

For $n=0$, the equation (3.12), become

$$
\left\{\begin{array}{c}
y_{1}(t)=A^{-1}\left[\frac{1}{v^{\alpha}} A\left[2 B_{0}\right]\right]=A^{-1}\left[\frac{1}{v^{\alpha}} A\left[2 y_{0}^{2}\left(\frac{t}{2}\right)\right]\right] \\
=A^{-1}\left[\frac{1}{v^{\alpha}} A\left[2\left(\frac{t^{\alpha}}{2^{\alpha} \Gamma(\alpha+1)}\right)^{2}\right]\right]=A^{-1}\left[\frac{1}{v^{\alpha}} A\left[\left(\frac{t^{2 \alpha}}{2^{2 \alpha-1} \Gamma^{2}(\alpha+1)}\right)\right]\right] \\
=A^{-1}\left[\frac{1}{v^{3 \alpha+2}} \frac{\Gamma(2 \alpha+1)}{2^{2 \alpha-1} \Gamma^{2}(\alpha+1)}\right]=\frac{\Gamma(2 \alpha+1)}{2^{2 \alpha-1} \Gamma^{2}(\alpha+1)} A^{-1}\left[\frac{1}{v^{3 \alpha+2}}\right] \\
=\frac{\Gamma(2 \alpha+1)}{2^{2 \alpha-1} \Gamma^{2}(\alpha+1)} \times \frac{t^{3 \alpha}}{\Gamma(3 \alpha+1)}
\end{array}\right.
$$

we choose $C=\frac{\Gamma(2 \alpha+1)}{2^{2 \alpha-1} \Gamma^{2}(\alpha+1)}$, so, we have :

$$
y_{1}(t)=C \frac{t^{3 \alpha}}{\Gamma(3 \alpha+1)},
$$

and,

$$
y_{1}\left(\frac{t}{2}\right)=C \frac{t^{3 \alpha}}{2^{3 \alpha} \Gamma(3 \alpha+1)},
$$


for $n=0$, the equation (3.12), become :

$$
\left\{\begin{array}{c}
y_{2}(t)=A^{-1}\left[\frac{1}{v^{\alpha}} A\left[2 B_{1}\right]\right]=A^{-1}\left[\frac{1}{v^{\alpha}} A\left[4 y_{0}\left(\frac{t}{2}\right) y_{1}\left(\frac{t}{2}\right)\right]\right] \\
=A^{-1}\left[\frac{1}{v^{\alpha}} A\left[4\left(\frac{t^{\alpha}}{2^{\alpha} \Gamma(\alpha+1)}\right)\left(C \frac{t^{3 \alpha}}{2^{3 \alpha} \Gamma(3 \alpha+1)}\right),\right]\right] \\
=A^{-1}\left[\frac{1}{v^{\alpha}} A\left[C\left(\frac{t^{4 \alpha}}{2^{4 \alpha-2} \Gamma(\alpha+1) \Gamma(3 \alpha+1)}\right)\right]\right] \\
=A^{-1}\left[\frac{1}{v^{5 \alpha+2}} \frac{C . \Gamma(4 \alpha+1)}{2^{4 \alpha-2} \Gamma(\alpha+1) \Gamma(3 \alpha+1)}\right]=\frac{C \cdot \Gamma(4 \alpha+1)}{2^{4 \alpha-2} \Gamma(\alpha+1) \Gamma(3 \alpha+1)} A^{-1}\left[\frac{1}{v^{5 \alpha+2}}\right] \\
=\frac{C \cdot \Gamma(4 \alpha+1)}{2^{4 \alpha-2} \Gamma(\alpha+1) \Gamma(3 \alpha+1)} \times \frac{t^{5 \alpha}}{\Gamma(5 \alpha+1)} .
\end{array}\right.
$$

The series solution is given by:

$$
y(t)=y_{0}(t)+y_{1}(t)+y_{2}(t)+y_{3}(t)+\ldots
$$

In particular case $\alpha=1$, then we obtain:

$$
\left\{\begin{array}{c}
y_{0}(t)=\frac{t}{\Gamma(2)}=t \\
y_{1}(t)=\frac{\Gamma(2 \alpha+1)}{2^{2 \alpha-1} \Gamma^{2}(\alpha+1)} \times \frac{t^{3 \alpha}}{\Gamma(3 \alpha+1)}=\frac{\Gamma(3)}{2^{1} \Gamma^{2}(2)} \times \frac{t^{3}}{\Gamma(4)}=\frac{t^{3}}{3 !} \\
y_{2}(t)=\frac{C \cdot \Gamma(4 \alpha+1)}{2^{4 \alpha-2} \Gamma(\alpha+1) \Gamma(3 \alpha+1)} \times \frac{t^{5 \alpha}}{\Gamma(5 \alpha+1)}=\frac{C \cdot \Gamma(5)}{2^{2} \Gamma(2) \Gamma(4)} \times \frac{t^{5}}{\Gamma(6)}=\frac{t^{5}}{5 !} .
\end{array}\right.
$$

Recall that,

$$
\Gamma(n+1)=n !, \quad \forall n \in \mathbb{N} .
$$

The exact solution when $\alpha=1$ is given by:

$$
y(t)=t+\frac{t^{3}}{3 !}+\frac{t^{5}}{5 !}+\ldots=\sinh (t) .
$$

Example 3.3. Consider the nonlinear delay differential equation of first $\operatorname{order}(n=2)$

$$
D^{\alpha} y(t)=1-2 y^{2}\left(\frac{t}{2}\right), \quad 0 \leqslant t \leqslant 1, \quad 1<\alpha \leqslant 2 .
$$

with initial condition

$$
y(0)=1, \quad y^{\prime}(0)=0 .
$$

Apply Aboodh transform to both side of (3.13), we get :

$$
A\left[D^{\alpha} y(t)\right]=A\left[1-2 y^{2}\left(\frac{t}{2}\right)\right] .
$$

Using definition (3.1) and initial condition :

$$
\begin{aligned}
& v^{\alpha} A[y(t)]-v^{\alpha-2} y(0)-v^{\alpha-3} y^{\prime}(0)=\frac{1}{v^{2}}-2 A\left[y^{2}\left(\frac{t}{2}\right)\right] \\
& A[y(t)]=\frac{1}{v^{2}}+\frac{1}{v^{2+\alpha}}-2 v^{-\alpha} A\left[2 y\left(\frac{t}{2}\right)\right] .
\end{aligned}
$$

Applying the inverse Aboodh transform to(3.14), we get :

$$
\begin{aligned}
& y(t)=A^{-1}\left[\frac{1}{v^{2}}\right]+A^{-1}\left[\frac{1}{v^{2+\alpha}}\right]-2 A^{-1}\left[v^{-\alpha} A\left[y^{2}\left(\frac{t}{2}\right)\right]\right] \\
& y_{0}(t)=A^{-1}\left[\frac{1}{v^{2}}\right]+A^{-1}\left[\frac{1}{v^{2+\alpha}}\right]=1+\frac{t^{\alpha}}{\Gamma(\alpha+1)} \\
& y_{0}\left(\frac{t}{2}\right)=1+\frac{t^{\alpha}}{2^{\alpha} \Gamma(\alpha+1)}
\end{aligned}
$$




$$
y_{n+1}=-2 A^{-1}\left[v^{-\alpha} A\left[B_{n}\right]\right]
$$

from equation (2.10), we have :

$$
\left\{\begin{array}{c}
B_{0}=f\left(y_{0}\right)=y_{0}^{2}\left(\frac{t}{2}\right)=\left(1+\frac{t^{\alpha}}{2^{\alpha} \Gamma(\alpha+1)}\right)^{2}=1+\frac{t^{\alpha}}{2^{\alpha-1} \Gamma(\alpha+1)}+\frac{t^{2 \alpha}}{2^{2 \alpha} \Gamma^{2}(\alpha+1)} \\
B_{1}=y_{1} f^{\prime}\left(y_{0}\right)=2 y_{0}\left(\frac{t}{2}\right) y_{1}\left(\frac{t}{2}\right) \\
B_{2}=y_{2}\left(\frac{t}{2}\right) 2 y_{0}\left(\frac{t}{2}\right)+\frac{1}{2} y_{1}^{2} . \\
\vdots
\end{array}\right.
$$

For $n=0$, the equation (3.15), become:

$$
\left\{\begin{array}{c}
y_{1}=-2 A^{-1}\left[v^{-\alpha} A\left[B_{0}\right]\right]=-2 A^{-1}\left[v^{-\alpha} A\left[1+\frac{t^{\alpha}}{2^{\alpha-1} \Gamma(\alpha+1)}+\frac{t^{2 \alpha}}{2^{2 \alpha} \Gamma^{2}(\alpha+1)}\right]\right] \\
=-2 A^{-1}\left[\frac{1}{v^{\alpha+2}}+\frac{1}{2^{\alpha-1} v^{2 \alpha+2}}+\frac{\Gamma(2 \alpha+1)}{2^{2 \alpha} v^{3 \alpha+2} \Gamma^{2}(\alpha+1)}\right] \\
=\frac{-2 t^{\alpha}}{\Gamma(\alpha+1)}-\frac{t^{2 \alpha}}{2^{\alpha-2} \Gamma(2 \alpha+1)}-\frac{t^{3 \alpha} \Gamma(2 \alpha+1)}{2^{2 \alpha-1} \Gamma^{2}(\alpha+1) \Gamma(3 \alpha+1)} .
\end{array}\right.
$$

The series solution is given by :

$$
\left\{\begin{array}{c}
y(t)=y_{0}(t)+y_{1}(t)+y_{2}(t)+y_{3}(t)+\ldots \\
=1+\frac{t^{\alpha}}{\Gamma(\alpha+1)}-\frac{2 t^{\alpha}}{\Gamma(\alpha+1)}-\frac{t^{2 \alpha}}{2^{\alpha-2} \Gamma(2 \alpha+1)}-\frac{t^{3 \alpha} \Gamma(2 \alpha+1)}{2^{2 \alpha-1} \Gamma^{2}(\alpha+1) \Gamma(3 \alpha+1)}+\ldots .
\end{array}\right.
$$

In particular case $\alpha=2$, then we obtain :

$$
\left\{\begin{array}{c}
y_{0}(t)=1+\frac{t^{2}}{\Gamma(3)}=1+\frac{t^{2}}{2 !}=1+\frac{t^{2}}{2} \\
y_{1}(t)=\frac{-2 t^{\alpha}}{\Gamma(\alpha+1)}-\frac{t^{2 \alpha}}{2^{\alpha-2} \Gamma(2 \alpha+1)}-\frac{t^{3 \alpha} \Gamma(2 \alpha+1)}{2^{2 \alpha-1} \Gamma^{2}(\alpha+1) \Gamma(3 \alpha+1)} \\
=-\frac{2 t^{2}}{2 !}-\frac{t^{4}}{2^{0} \Gamma(5)}-\frac{t^{6} \Gamma(5)}{2^{3} \Gamma^{2}(3) \Gamma(7)}=-t^{2}-\frac{t^{4}}{4 !}-\frac{3 t^{6}}{4 \times 6 !} .
\end{array}\right.
$$

The exact solution when $\alpha=2$ is given by :

$$
y(t)=y_{0}(t)+y_{1}(t)+\ldots=1-\frac{t^{2}}{2}+\frac{t^{4}}{4 !}-\ldots=\cos (t) .
$$

\section{Conclusion}

This study aims to propose an efficient algorithm for the solution of nonlinear fractional equations. The adomian decomposition method has been recognized as a powerful technique to solve many nonlinear differential equations. In this work, a combined method which groups together the transform (2.1) and the adomian decomposition are discussed to find an explicit approximate solution for fractional Delay Differential Equations (DDEs).

\section{References}

[1] M. Elarbi Benattia, K. Belghaba, Application of the Galerkin method with Chebyshev polynomials for solving the integral equation, J. Comput. Sci. Comp. Math., DOI: 10.20967/jcscm.2017.03.008.

[2] M. Elarbi Benattia, K. Belghaba, The analytical solution Of Telegraph equation Of space-fractional order derivative by the Aboodh transform Method, Int. J. Anal. Appl., 18(2), (2020), 243-253.

[3] Ebimene, J. Mamadu, Ignatius N. Njoseh, Solving delay differential equationns by Elzaki transform method, Boson J. Modern Phys., (BJMP)3-1 (2017).

[4] H. Eltayeb, E. Abdeldaiem, Sumudu decomposition method for solving fractional delay differential equations, Res. Appl. Math,, 1 (2017), 1-13.

[5] F. Ismail, R.A. Al-Khasawneh, A.S. Lwin and M. Suleiman, Numerical treatment of delay differential equations by Runge-Kutta method using hermite interpolation, Matematika, 18(2), (2002), 79-90.

[6] S.T. Demiray, H. Bulut and F.B.M. Belgacem, Sumudu transform method for analytical solutions of fractional type ordinary differential equations, J. Math. Prob. Eng., (2015). http://dx.doi.org/10.1155/2014/131690.

[7] H. Smith, An Introduction to Delay Differential Equations with Applications to the Life Sciences, Springer, New York, NY, USA, 2011.

[8] S.T. Mohyud-Dina and A. Y1ldırım, Variational iteration method for delay differential equations using He's polynomials, Z. Naturforsch. 65a (2010), 1045-1048

[9] Abas.I. Khlaif. Approximate solutions for delay differential equations of fractional order. Thesis(B.Sc mathematics/ College science/ Al -Nahrain university 2012). 